
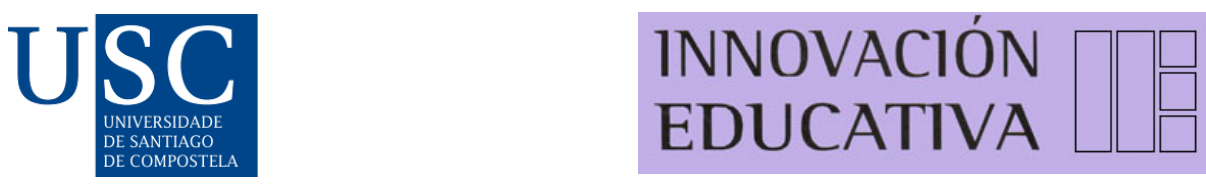

Innovación Educativa, (31), 2021. ISSN-e: 2340-0056

https://doi.org/10.15304/ie.31.7664

Artigos

\title{
INSTRUMENTOS MUSICALES 2.0: UN PROYECTO INTERCENTRO PARA EL DESARROLLO DE LA COMPETENCIA DIGITAL $Y$ ARTÍSTICA EN EDUCACIÓN PRIMARIA
} MUSICAL INSTRUMENTS 2.0: AN INTERCENTER PROJECT FOR THE DEVELOPMENT
OF DIGITAL AND ARTISTIC COMPETENCE IN ELEMENTARY EDUCATION

\author{
Salvador Oriola Requena, a ${ }^{1,}$ \\ ${ }^{1}$ Facultad de Educación, Universitat de Barcelona, España \\ a salvaoriola@ub.edu
}

Recibido: 26/04/2021; Aceptado: 01/11/2021

\section{Resumen}

En el presente artículo se expone y se analiza la realización de un proyecto telecolaborativo realizado por centros educativos de diferente índole (escuela de primaria, conservatorio y escuelas de música) para el aprendizaje de los instrumentos musicales. El principal objetivo es conocer si la combinación del uso de las TIC (herramientas 2.0) junto con el trabajo por proyectos influye en el desarrollo competencial del alumnado de primaria. Para ello se ha utilizado una metodología mixta a través de diferentes instrumentos: análisis de resultados académicos, entrevistas semiestructuradas y observación participante. Los resultados obtenidos reflejan que la realización de este tipo de proyectos en red no solo mejora la adquisición competencial, sino también el clima social de las clases y la motivación de los participantes.

Palabras clave: TIC; proyectos; educación primaria; competencias; instrumentos musicales.

\begin{abstract}
This article presents and analyses a telecollaborative project carried out by different kinds of schools (elementary school, conservatory and music schools) to learn about musical instruments. The main objective is to know if the combination of the use of ICT (2.0 tools) with project work influences the development of competences of primary school students. For this, a mixed methodology has been used through different instruments: comparison of academic results, semi-structured interviews and participant observation. The results obtained reflect that carrying out the project not only improves academic results, but also motivation and the work environment.
\end{abstract}

Keywords: ICT; projects; primary education; competences; musical instruments. 


\section{INTRODUCCIÓN}

El uso cotidiano de dispositivos móviles, la hiperconectividad o el acceso a todo tipo de información, desde cualquier sitio en cualquier momento, son solo algunos ejemplos de cómo las tecnologías de la información y comunicación (TIC) han influido en la transformación del modo de vivir y relacionarse las personas. Todo ello se enmarca en lo que se conoce como realidad VUCA, acrónimo anglosajón formado por las iniciales de las palabras Volatility, Uncertainty, Complexity y Ambiguity, es decir, vivimos en un mundo cambiante, incierto, complejo y ambiguo en el que de acuerdo con Marina (2015), la educación deberá reformularse constantemente para adaptarse a este contexto inédito y poder así formar futuros ciudadanos que puedan afrontar con garantías de éxito su vida tanto personal como profesional.

Las administraciones educativas, el profesorado, las familias y la comunidad educativa en general, son conscientes del dinamismo de las sociedades actuales y cómo los intereses y necesidades del alumnado han cambiado significativamente en las últimas décadas, por esta razón el sistema educativo actual ha virado hacia un modelo competencial basado en el aprendizaje transversal, transferible y continuo que vaya más allá de la memorización de conceptos y que permita resolver al alumnado problemas de su vida cuotidiana (Monarca et al, 2016). De acuerdo con la legislación educativa vigente (Departamento de Educación de la Generalitat de Catalunya, 2015) todo ello va en consonancia con las referencias internacionales expuestas por la CEOE y la Unión Europea, siguiendo los últimos avances de disciplinas como las neurociencias, la pedagogía o la psicología.

En este contexto, la competencia digital en la educación obligatoria se ha establecido como una de las competencias transversales con más protagonismo, puesto que se trabajará desde todas las áreas e irá más allá de un mero conocimiento de instrumentos y aplicaciones digitales (PérezEscoda et al. 2016).

Hasta principios del siglo XXI la educación digital, conocida en las escuelas como informática, era unidireccional y consistía en aprender a usar unos softwares determinados (editores de texto, de imágenes, de audio, etc.), pero en las últimas dos décadas, factores como la bidireccionalidad, la velocidad con la que se genera información o el alto grado de obsolescencia han modificado sustancialmente el aprendizaje y uso de las TIC tanto en las aulas como fuera de estas, potenciando un procesamiento de información rápido, superficial y algunas veces disperso, lo cual provoca que el alumnado, nativo digital, se aburra fácilmente con las metodologías tradicionales (Keengwe y Georgina, 2013). Tal y como afirman Muntaner et al. (2020), en las escuelas actuales se necesitan metodologías activas para aumentar el ritmo de estimulación y la motivación entre el alumnado y contribuir así a una mejora en el flujo de ideas y la creatividad.

Ante esta nueva situación, la educación contemporánea deberá promover una alfabetización digital multimediática que permita al alumnado interactuar, crear y compartir contenidos de todo tipo, usando múltiples medios: sonoros, visuales, hipertextuales, simuladores, juegos, redes sociales, etc. (Dorantes et al., 2018). Todo ello coincide con los postulados expuestos por SánchezVera et al. (2019), según los cuales, las TIC en las aulas, además de dar a conocer instrumentos y aplicaciones digitales, deberán contribuir a una renovación pedagógica basada en metodologías activas que modifiquen los roles tradicionales de profesor y alumno, y sirvan como recurso para la implementación de metodologías activas como por ejemplo aprendizaje por proyectos, el trabajo en equipo o los proyectos 2.0, también conocidos como telecolaborativos. 
El binomio existente entre la música y las nuevas tecnologías puede aprovecharse en la educación obligatoria para fomentar este tipo de metodologías y desarrollar al mismo tiempo diferentes competencias como la digital o la artística en alumnado tanto de primaria como secundaria, tal y como demuestran algunas propuestas innovadoras sobre Música y TIC (Giráldez, 2015; Masdeu, 2017; Monreal et al., 2015), las cuales se han incrementado y difundido exponencialmente en los últimos años.

En nuestro caso presentamos un proyecto telecolaborativo entre centros educativos de distinta índole (escuela de primaria, conservatorio de música y escuelas de música), el cual consiste en la creación de un recurso didáctico 2.0 para conocer y difundir información básica sobre los instrumentos musicales de la orquesta. Con la realización del proyecto se pretende mejorar tanto la competencia digital como artística de los alumnos participantes de ciclo superior de primaria. Todo ello se enmarca dentro de lo que se conoce como enfoque comunitario de inclusión educativa, basado en un trabajo en red en el que los centros participantes colaboran, indagan y cooperan para alcanzar metas compartidas. De acuerdo con las palabras de Parrilla et al. (2018, p. 67):

Es este conjunto de situaciones y circunstancias en interacción y de manera fluida, lo que permite abrir una vía de escape ante el aislamiento que tradicionalmente ha caracterizado a las escuelas, y crear espacios compartidos de participación, implicación y corresponsabilidad para la mejora escolar y social.

\section{DESCRIPCIÓN DEL PROYECTO INSTRUMENTOS MUSICALES 2.0}

El origen del proyecto Instrumentos musicales 2.0 nace por la necesidad, en el contexto de educación primaria, de crear un material didáctico atractivo para el aprendizaje de los instrumentos musicales. Existe abundante y variado material didáctico sobre dicho tema, como: libros de texto con audios, páginas web, videos en YouTube, etc., pero no se encontró ninguno que se adaptase en su totalidad a las necesidades e intereses de los alumnos y alumnas de primaria. Ante esta situación se vio la necesidad de crear un recurso didáctico de fácil acceso que reuniera en un mismo sitio web, una parte teórica sobre cada instrumento de la orquesta complementada con un video, para que la persona que accediera pudiera, además de conocer aspectos teóricos, ver y escuchar de forma individual cada instrumento musical. Para ello se propuso llevar a cabo un proyecto telecolaborativo entre centros de régimen general (escuela pública) y centros de régimen especial (escuelas de música y conservatorio profesional). Cada centro se encargaría de elaborar una parte específica del proyecto y obtener así un material didáctico final del cual se pudieran beneficiar tanto las partes participantes como cualquier docente o alumnado interesado en el tema.

Todo el proceso de elaboración fue llevado a cabo con herramientas 2.0, con las que tanto el alumnado como el profesorado involucrado, interactuaron y aprendieron haciendo, consiguiendo así que el alumnado fuera el epicentro de su propio aprendizaje a través, por ejemplo, de la creación de contenidos digitales, la grabación y edición de los videos de los instrumentos musicales o el montaje de una exposición a través del uso de códigos QR. Todo ello conllevó la aplicación de metodologías activas, productivas y transferibles para un aprendizaje competencial basado en sacar el máximo rendimiento didáctico a las posibilidades que ofrecen las TIC y dejar a un lado la reproducción de modelos tradicionales basados en la exposición de contenidos (Céspedes Ventura y Ballesta Pagán, 2018). 


\subsection{Diseño}

Como en cualquier proyecto, la primera acción fue debatir con los alumnos y las alumnas de primaria, la importancia que tenía el aprendizaje de los instrumentos musicales en la asignatura de música y cómo se podía llevar a cabo de una forma atractiva. Para ello se hizo un brainstorming del cual salió la idea de ponerse en contacto con alumnos y alumnas de escuelas de música y conservatorios para que les enseñaran y explicaran las características tanto físicas como sonoras de sus instrumentos musicales y a partir de ahí poder crear una descripción detallada sobre cada uno de ellos.

Así pues, nos pusimos en contacto con profesores y profesoras de distintos centros musicales, los cuales aceptaron la invitación para participar con sus alumnos y alumnas en el proyecto. Dicho profesorado se encargó de grabar diferentes vídeos de cada instrumento de la orquesta tocado por su alumnado. Estos videos se grabaron con dispositivos móviles (teléfonos y IPads) y se subieron a una carpeta Google Drive, a la cual todo el profesorado y alumnado implicado en el proyecto tenían acceso.

Una vez fueron recopilados los videos, los alumnos y las alumnas de la escuela de primaria se encargaron de escoger democráticamente y editar los videos que presentaban una mejor calidad tanto artística como de imagen y sonido. Posteriormente los videos editados fueron subidos a un canal de YouTube creado por el alumnado para la ocasión.

Es cierto que los profesores y las profesoras de instrumento del conservatorio y las escuelas de música se hubieran podido grabar a ellos mismos -para conseguir un mayor nivel artístico-, pero se decidió que los intérpretes fueran los alumnos y las alumnas de grado elemental y profesional (de 7 a 16 años) por una doble razón: por una parte, debían prepararse una pieza musical para ser interpretada públicamente en el proyecto -lo cual motivó significativamente a estos alumnos y alumnas- y por otra parte, se pensó que los niños y niñas de primaria a los que van destinados los videos se sentirían mucho más identificados con materiales audiovisuales donde los protagonistas tuvieran una edad similar.

Los alumnos y alumnas de ciclo superior de primaria, coordinados por el maestro de música, crearon una página web con Wordpress, estructurada a partir de la clasificación tradicional de los instrumentos musicales y cuyo contenido consistía en una sección específica para cada uno de los instrumentos de la orquesta. En las clases de música y TIC se crearon los contenidos digitales a partir de la exposición realizada por los alumnos y las alumnas que habían grabado el video y complementado con la búsqueda y recopilación de información en libros de texto e Internet. La información de cada instrumento (descripción, fotos, partes, características...) se guardaba en diferentes Google Docs para que posteriormente fuera revisada por los profesores implicados. Cuando todo estaba revisado y bien escrito -con un contenido y vocabulario adecuado para el nivel de primaria- los mismos alumnos y alumnas de primaria se encargaron de incorporar toda la información revisada a la web. Al tratarse de una escuela de Catalunya, cuya lengua cooficial es el catalán, toda la web se escribió en dicha lengua. Por esta razón en las asignaturas de inglés y lengua castellana, con la ayuda de los respectivos docentes, se tradujeron del catalán todos los textos en castellano e inglés, y fueron agregados a la web para que esta fuera trilingüe y tuviera una mayor proyección.

Finalmente, para acabar la web se insertaron en las secciones correspondientes, los videos que habían sido grabados por los alumnos y las alumnas de los centros de régimen especial y que se 
habían subido al canal de YouTube. Tanto imágenes como vídeos fueron insertados por los alumnos de primaria a través de los enlaces generados para tal fin.

Con la web finalizada, el siguiente paso fue el diseño de una exposición virtual. Esta exposición fue realizada en la escuela de primaria, el conservatorio y las escuelas de música. Primero se creó un código QR para cada instrumento y para cada familia instrumental. Con el escáner de estos códigos, a través de dispositivos móviles, se accede a los contenidos (videos, textos y fotos) albergados en la web. Después de la impresión de los códigos QR, se imprimieron dibujos de cada instrumento que fueron coloreados, recortados y plastificados por el alumnado, con el objetivo de conocer mejor sus características físicas (tamaño, forma, color...). Finalmente se montó la exposición que consistía en un mural en el que cada dibujo del instrumento iba junto a su código QR. De esta manera las personas visitantes a la exposición virtual, además de ver el dibujo físico de cada instrumento como ocurriría en cualquier exposición, podían acceder y ver, a través de un dispositivo móvil, toda la información digital vinculada a cada instrumento que estaba albergada en la web: descripciones, fotos y videos (para mayor detalle sobre todo proyecto se recomienda ver Oriola Requena et al., 2014).

\section{MÉTODO}

La presente investigación consiste en un estudio descriptivo cuya metodología es de tipo mixta (cualitativa-cuantitativa). A partir del análisis de información de diferente índole, recopilada durante el proceso de elaboración del proyecto descrito, se pretende conocer el desarrollo de la competencia digital y artística por parte de los alumnos de primaria, así como la valoración de los participantes (alumnos y profesores) y la funcionalidad de la web realizada.

\subsection{Muestra participante}

Un grupo experimental de 50 alumnos/as de una escuela pública de Catalunya situada en una población cercana a Barcelona (Centro 1). De este grupo, 23 eran niños (46\%) y 27 niñas (54\%), cuya media de edad era de 10.59, con una desviación estándar de .526. Estos participaron activamente en la elaboración del proyecto descrito.

Un grupo control de 50 alumnos/as de la misma escuela (Centro 1), 29 niños (58\%) y 21 niñas (42\%), cuya media de edad era 10.87, con una desviación estándar de .876.

Un grupo de 20 alumnos/as de escuelas de música y conservatorio, 10 niños (50\%) y 10 niñas (50\%), cuya edad media era 14,74, con una desviación estándar de 1.486.

Y finalmente, 4 profesores/as, uno por cada centro educativo implicado (una escuela pública, un conservatorio y 2 escuelas de música), 2 mujeres y 2 hombres, cuya media de edad era 35.23, con una desviación estándar de 5.67, se encargaron de realizar una observación participante.

\subsection{Instrumentos para la recopilación de información y datos}

La recopilación y el análisis de información se ha llevado a cabo con los siguientes instrumentos:

Análisis y comparación de los resultados académicos de la competencia digital y artística entre el grupo control y el grupo experimental de alumnado de ciclo superior de primaria (Centro 1). 
Los resultados académicos de la competencia digital y artística están conformados por la media obtenida a partir de la valoración de unos ítems preestablecidos relacionados con las tareas que se llevan a cabo. En el caso de la competencia digital se valora el conocimiento y uso de las aplicaciones utilizadas para la edición de texto, imagen y video, la búsqueda y la selección de información digital, así como el buen uso de entornos digitales de colaboración. En la competencia artística se valora el aprendizaje de los instrumentos musicales, así como sus características tanto físicas como sonoras. Con la ayuda del software Excel en su versión 2016, se han calculado datos como porcentajes, promedios, desviaciones, etc.; asimismo, se ha realizado un análisis de varianza (ANOVA) para conocer si existen diferencias estadísticamente significativas entre ambos grupos. La comparación de los resultados académicos se llevó a cabo al final de la realización del proyecto.

Una observación participante sistemática de todo el proceso y de los agentes implicados. Todas las observaciones, tanto del proceso de elaboración como de los resultados, fueron registradas por el profesorado en un bloc de notas siguiendo un guion prestablecido y unas escalas de estimación. De acuerdo con dicho guion, los docentes encargados tenían que fijarse, comentar y puntuar unos indicadores preestablecidos relacionados con el rendimiento, eficacia, colaboración, uso de las TIC, motivación e implicación de los alumnos y las alumnas, clima social y de trabajo, etc. En palabras de Peña (2011), la participación activa del observador permite conocer al detalle las expectativas, intereses y actitudes de la muestra participante, de ahí su uso en nuestra investigación.

Entrevistas semiestructuradas a una muestra aleatoria de 20 alumnos y alumnas del grupo experimental. Para ello se elaboró un guion estructurado en torno a diferentes ítems relacionados con la participación en el proyecto Instrumentos musicales 2.0. Para el diseño de las entrevistas, se empleó el juicio de expertos a través de la validación por 6 jueces de los ítems propuestos. Todos ellos son profesores, especialistas en educación musical y TIC, de diferentes escuelas de primaria y universidades. Realizada la valoración de los expertos se incluyeron y modificaron algunos indicadores y seguidamente, se procedió a la realización de las entrevistas a un grupo aleatorio de la muestra total. A los entrevistados se les formularon preguntas abiertas como por ejemplo ¿qué opinas del proyecto? ¿te ha gustado? ¿te ha servido para algo? ¿qué mejorarías? ¿has aprendido nuevos conocimientos? ¿cuáles? De acuerdo con Fàbregues, et al. (2016), en una investigación educativa, como es el caso, este tipo de preguntas abiertas crea un clima cercano lo que posibilita mayor flexibilidad y matices en las respuestas de la muestra. Dichas entrevistas fueron registradas y posteriormente se transcribieron a un procesador de texto. Para el tratamiento de la información transcrita se optó por la categorización, a través del programa informático Atlas-ti Qualitative Data Analysis 7, que facilita el análisis cualitativo de datos. Procesada la información mediante dicho programa, se obtuvieron dos categorías estructurales: valoración del proceso y el desarrollo de competencias.

\subsection{Procedimiento}

Para la realización del proyecto y la consiguiente investigación se estableció un contacto inicial con los equipos directivos de los diferentes centros participantes para informales sobre todo el proceso y la planificación temporal. Aceptada la propuesta por parte de los centros educativos y los profesores implicados, se pasó a informar a las familias de los alumnos participantes. Al tratarse de una muestra formada por menores de edad, se facilitó a sus progenitores un documento informativo acerca de la investigación y la autorización para su participación de forma voluntaria y anónima. 
Obtenido el consentimiento informado, se pasó a la realización del proyecto y la investigación. La recopilación de datos se llevó a cabo mediante códigos de identificación para preservar el anonimato y la confidencialidad de los resultados, siguiendo en todo momento los condicionantes del Informe Belmont y el Código de Buenas Prácticas en Investigación, de la universidad a la que pertenecen los investigadores.

\section{RESULTADOS}

El alumnado participante en el proyecto Instrumentos musicales 2.0 (grupo experimental) obtuvo en las calificaciones de la competencia digital una nota media de 8.51 con una desviación estándar de .976, por otro lado, la nota media del alumnado que conformó el grupo control fue de 6.54 con una desviación estándar de 1.45. De acuerdo con los resultados del análisis de varianza ANOVA (F $\left.(3.93)=63.1 ;{ }^{*} \mathrm{p}<0.01\right)$ existen diferencias estadísticamente significativas entre grupos por lo que respecta al desarrollo de la competencia digital.

En cuanto a la competencia artística la nota media del grupo experimental fue de 7.798 con una desviación estándar de 1.18 mientras que la nota media del grupo control fue de 6.734 con una desviación estándar de 1.328. El análisis de varianza ANOVA ( F (3.93) $=17.46 ;{ }^{*} \mathrm{p}<0.01$ ) confirma que existen diferencias estadísticamente significativas entre grupos.

Ambos resultados reflejan que la participación activa por parte del alumnado de ciclo superior de primaria (Centro 1) en el proyecto Instrumentos musicales 2.0 influyó positivamente en el desarrollo tanto de la competencia digital como de la competencia artística.

Los datos recopilados a través de la observación participante, realizada por un profesor/a responsable de cada centro educativo implicado (4 profesores/as en total), confirman que tanto el diseño del proyecto como su realización a través de herramientas 2.0 suscitó una gran motivación por parte de todos los alumnos y las alumnas participantes, tanto de primaria como de los centros musicales, así como de los docentes. Los cuatro profesores/as coinciden en señalar que desde el principio hubo una gran implicación por parte de todos los participantes, lo cual repercutió positivamente no solo en los resultados obtenidos, sino también en el clima de trabajo y en la cooperación entre el alumnado. En el alumnado de primaria, las escalas de estimación relativas a la motivación y el clima de trabajo aportan valores superiores al 8.5. Las escalas de estimación sobre el desarrollo competencial se sitúan en torno al 8. Por otro lado, en el alumnado de escuelas de música y conservatorios la escala de estimación relativa a la motivación es de 8.6. Como puntos débiles se observó que la elaboración y la puesta en marcha de un proyecto de tal magnitud requiere de una gran dedicación, preparación y coordinación por parte del profesorado, lo cual implica más carga de trabajo fuera del horario lectivo. También apuntan la complicación de encontrar franjas horarias comunes para reunirse el profesorado de diferentes tipologías de centros (escuelas de primaria y centros de régimen especial), los cuales tienen horarios totalmente diferentes.

Por otro lado, el análisis de las entrevistas semiestructuradas realizadas a 20 alumnos/as reflejan una valoración muy positiva, tal y como se puede comprobar en afirmaciones como:

"Con la participación en este tipo de proyectos el tiempo ha pasado muy rápido porque las clases han sido divertidas y amenas" (Alumno entrevistado 5).

"Es muy original y aprendes cosas nuevas cuando estás en contacto con alumnos de tu edad que son de otras partes del mundo" (Alumno entrevistado 3).

"Ojalá pudiéramos hacer más proyectos como este en las otras asignaturas" (Alumno entrevistado 7). 


\footnotetext{
"Parecía que no estuviéramos en clase ya que cada uno sabía lo que tenía que hacer y cuando acababa ayudaba a sus compañeros" (Alumno entrevistado 16).
}

En cuanto al aprendizaje y el desarrollo de competencias, también existe una mayoría de entrevistados que autoperciben haber mejorado sus conocimientos y sus capacidades por lo que respecta a las TIC y a los instrumentos musicales:

"Ahora sí que sé qué es un fagot, cuáles son sus partes y cómo suena" (Alumno entrevistado 12).

"Trabajar telemáticamente con otros alumnos nos ha ayudado a aprender cómo hacer trabajos en grupo sin tener que quedar. Cada uno desde su casa" (Alumno entrevistado 20).

"He aprendido a editar videos y subirlos a YouTube, cosa que tenía muchas ganas de aprender" (Alumno entrevistado 9).

Como puntos de mejora, algún alumno/a entrevistado remarca la falta de tiempo para la realización de algunas tareas determinadas:

"Nos faltó tiempo para acabar de editar bien los videos" (Alumno entrevistado 15).

"Hubo poco tiempo para buscar información y hacer la descripción de los instrumentos musicales" (Alumno entrevistado 16).

\section{DISCUSIÓN Y CONCLUSIONES}

A raíz de los resultados expuestos y coincidiendo con otros estudios (Carrió, 2007; Méndez y Delgado, 2016) se puede concluir que las herramientas 2.0 y los teleproyectos entre centros educativos de diferente índole son un complemento para la labor docente y pueden contribuir de forma positiva en la implementación de nuevas metodologías y escenarios de aprendizaje. El objetivo principal de todo ello no es otro que la mejora de la motivación y el desarrollo competencial de los alumnos y las alumnas, nativos digitales, a través de un aprendizaje significativo y productivo sobre el buen uso de las TIC, tal y como ha ocurrido a lo largo de todo el proyecto Instrumentos musicales 2.0.

En nuestro caso, la telecolaboración, al eliminar el factor de la presencia física, ha permitido ensanchar la asignatura de música de la escuela de primaria a través de la colaboración y cooperación con alumnos y profesores de centros especializados en música como son conservatorios y escuelas de música. Todo ello coincide con postulados como los de Berbel y Díaz Gómez (2014) o Parrilla et al. (2018), según los cuales, trabajar con otras instituciones a través de las redes, planteando con ellos actividades y tareas contribuirá al desarrollo de un enfoque metodológico basado en aprender haciendo y aprender interactuando, tal y como ha corroborado la mejora competencial de los alumnos y las alumnas participantes, así como la opinión tanto de profesores como de alumnos.

Sin embargo, para el buen funcionamiento y el éxito de todo proyecto telecolaborativo será imprescindible una buena formación y organización por parte de los docentes implicados (Gutiérrez Martín, 2008). En este sentido será necesario contar con un grupo de docentes actualizados de forma permanente y continua en cuanto al uso de las TIC se refiere. Esto no significa solamente estar a la última en herramientas digitales, sino también saber escoger entre todas las existentes, aquellas que más se adapten a las características del proyecto que se quiera llevar a cabo, para sacarles el máximo provecho y poder así rentabilizar el tiempo de trabajo. Por ejemplo, en nuestro proyecto la organización y etiquetación en carpetas Google Drive de todo el material 
que se iba elaborando, junto con una distribución definida y clara de las diferentes tareas que cada alumno/a y profesor/a debía desempeñar, contribuyeron en todo momento al buen devenir del proyecto. En España el profesorado de música en todos los niveles está cada vez más al día con el uso de las TIC dentro de las aulas, pero tal y como comentan Calderón-Garrido et al. (2020) o Díez Latorre (2018), aún queda mucho camino por recorrer.

Además del proceso de elaboración del proyecto en el que se ha conseguido crear un clima de trabajo muy favorable que ha repercutido positivamente tanto en la motivación como en los resultados académicos de los participantes, también cabe destacar el producto final obtenido en forma de página web. Los más de dos millones de visitas en apenas 6 años por gente de diferentes partes del mundo es un indicador significativo del interés que ha suscitado el contenido didáctico elaborado.

Los resultados obtenidos en la presente investigación van en la línea de otros estudios como los de Azorín (2014) o Cruz (2016), según los cuales, un buen uso de las TIC como recurso didáctico en las aulas puede incentivar: metodologías participativas en ambientes que generan un clima favorable y motivador para el aprendizaje autónomo; el logro de los objetivos propuestos en un alto porcentaje; o la consecución de evaluaciones positivas con respecto al logro de adquisición y desarrollo de competencias. Sin embargo y pese a las coincidencias citadas, en el caso que nos ocupa se debe ser cauto con los buenos resultados obtenidos puesto que se trata de un caso concreto con un número limitado de muestra por lo que no se pueden extraer conclusiones categóricamente generalizables, para ello y como futura línea de investigación cabría realizar un estudio con una muestra más amplia y grupos de diferentes escuelas y procedencias.

Para terminar, vivimos en un nuevo escenario global en el que el acceso a la información es infinito, los cambios sociales son incesantes o la interconexión entre personas no cede, por esta razón los docentes y educadores tenemos que formarnos, renovarnos y aprovechar todos estos nuevos recursos digitales para formar a futuros ciudadanos que sean competentes y críticos con lo que respecta al uso de las TIC. Esta teoría es clara, pero se necesita de propuestas prácticas y funcionales como la expuesta aquí con el fin de tener modelos referenciales que puedan ser transferibles y adaptables a otros contextos (Perines, 2018). Cuantas más investigaciones y experiencias didácticas se lleven a cabo y se difundan sobre la inclusión de las TIC en las aulas más amplio será el abanico de recursos digitales de los que dispondrán los docentes para el beneficio de sus alumnos y alumnas.

\section{REFERENCIAS}

Azorín, C. M. (2014). ¿Cómo motivar a los nativos digitales para la mejora del proceso de enseñanzaaprendizaje? El universo Wix en el aula. En C. J. Gómez y A. Escarbajal (Eds.), Calidad e Innovación en Educación Primaria (pp. 37-48). Servicio de Publicaciones de la Universidad de Murcia.

Berbel, N. y Díaz Gómez, M. (2014). Educación formal y no formal. Un punto de encuentro en educación musical. Aula Abierta, 42(1), 47-52. https://doi.org/10.1016/S0210-2773(14)70008-3

Calderón-Garrido, D., Gustems-Carnicer, J. y Carrera, X. (2020). Digital technologies in music subjects on primary teacher training degrees in Spain: Teachers' habits and profiles. International Journal of Music Education, 38(4), 613-624. https://doi.org/10.1177/0255761420954303

Carrió, M. L. (2007). Ventajas del uso de la tecnología en el aprendizaje colaborativo. Revista Iberoamericana de Educación, 41(4), 1-10. 
Céspedes Ventura, R. y Ballesta Pagán, J. (2018). Acceso, uso y actitud de la tecnología en las escuelas de Educación Primaria. Aula Abierta, 47(3), 355-364. https://doi.org/10.17811/rifie.47.3.2018.355-364

Cruz, R. (2016). Buenas prácticas que desarrollan la competencia mediática en entornos socioeducativos. Píxel-Bit. Revista de Medios y Educación, (48), 97-113. https://doi.org/10.12795/pixelbit.2016.i48.07

Departamento de Educación de la Generalitat de Catalunya. (2015). Decreto 119/2015, del 23 de junio, de ordenación de las enseñanzas de la educación primaria en Catalunya.

Díez Latorre, N. (2018). La formación en TIC de los pedagogos de música. Análisis de la situación en las Enseñanzas Superiores de Música. Revista Electrónica de LEEME, (42), 31-51. https://doi.org/10.720 3/LEEME.42.13067

Dorantes, P., Acosta, A., y Rodríguez, A. (2018). Alfabetismo digital, saberes digitales, competencias informacionales y capital digital: tensiones entre la polisemia y la homogeneidad. En E. Ruiz-Velasco, J. Bárcenas y J. A. Domínguez (Eds.), Construcción social de una cultura digital educativa (pp. 75-84). Somece.

Fàbregues, S., Meneses, J., Rodríguez-Gómez, D. y Paré, M. H. (2016). Técnicas de investigación social y educativa. Editorial UOC.

Giráldez, A. (2015). (Coord.). De los ordenadores a los dispositivos móviles. Propuestas de creación musical y audiovisual. Editorial Graó.

Gutiérrez Martín, A. (2008). Las TIC en la formación del maestro. "Realfabetización” digital del profesorado. Revista interuniversitaria de formación del profesorado, 22(3), 191-206. https://www.redalyc.org/pdf /274/27418813011.pdf

Keengwe, J., y Georgina, D. (2013). Supporting Digital Natives to Learn Effectively with Technology Tools. International Journal of Information and Communication Technology Education (IJICTE), 9(1), 51-59. $\underline{\mathrm{h}}$ ttps://doi.org/10.4018/jicte.2013010105

Marina, J. A. (2015). Despertad al diplodocus: Una conspiración educativa para reformar la escuela..., y todo lo demás. Ariel.

Masdeu, E. (2017). La revolució digital a les aules de música. Pagès Editors.

Méndez, J. M., y Delgado, M. (2016). Las TIC en centros de Educación Primaria y Secundaria de Andalucía. Un estudio de casos a partir de buenas prácticas. Digital Education Review, (29), 134-165.

Monarca, H., Simón, C., Rappoport, S., y Echeita, G. (2016). Política y cambio en educación: el caso de las competencias básicas en España. Ensaio: Avaliação e Políticas Públicas em Educação, 24(93), 968-989. https://doi.org/10.1590/S0104-40362016000400009

Monreal, I. M., Giráldez, A. y Gutiérrez, A. (2015). Use and curricular integration of the digital interactive whiteboard in the music classroom: A single case study in Segovia. Multidisciplinary Journal of Educational Research, 5(1), 82-104. https://doi.org/10.4471/remie.2015.04

Muntaner, J. J., Pinya, C. y Mut, B. (2020). El impacto de las metodologías activas en los resultados académicos. Profesorado. Revista de Currículum y Formación de Profesorado, 24(1), 96-114. https://doi.org/10.308 27/profesorado.v24i1.8846

Oriola Requena, S., Nogueras Tarrero, L. y Gimeno Torres, F. (2014). Proyecto 2.0 sobre los instrumentos musicales a partir de la colaboración entre Escuelas y Conservatorios de Música, Centros de Primaria y músicos profesionales. En M. Serrat y E. Molina, Actas del III Congreso Nacional de Educación e Investigación Musical (CEIMUS) (pp. 254-262). Recuperado de https://enclavecreativa.com/wp-conte nt/uploads/2019/09/Actas-CEIMUS-III.pdf

Parrilla, A., Sierra, S. y Fiuza, M. (2018). Lecciones esenciales sobre el trabajo en red interescolar. Profesorado. Revista de Currículum y Formación de Profesorado, 22(2), 51-69. https://doi.org/10.30827/profesora do.v22i2.7714 
Peña, B. (2011). Métodos científicos de observación en Educación. Visión Libros.

Pérez-Escoda, A., Castro-Zubizarreta, A., y Fandos-Igado, M. (2016). La competencia digital de la Generación Z: claves para su introducción curricular en la Educación Primaria. Comunicar, 24(49), 71-80. https:/ /doi.org/10.3916/C49-216-07

Perines, H. (2018). ¿Por qué la investigación educativa no impacta en la práctica docente? Estudios sobre educación, 34, 9-27. https://doi.org/10.15581/004.34.9-27

Sánchez-Vera, M. M., Solano-Fernández, I. M. y Recio-Caride, S. (2019). El storytelling digital a través de vídeos en el contexto de la educación infantil. Píxel-BIT: Revista de Medios y Educación, (54), 165-184. https://doi.org/10.12795/pixelbit.2019.i54.09 